\title{
Anemia y patología digestiva
}

\section{Anemia and digestive diseases}

\section{Anemia e patologia digestiva}

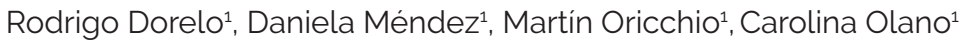

RESUMEN

Cada vez más los pacientes diagnosticados con anemia son referidos al gastroenterólogo para su evaluación. La necesidad de realizar un adecuado planteo clínico y una correcta interpretación de las pruebas de diagnóstico ha motivado la revisión de este tema.

Varios trastornos gastroenterológicos, con frecuencia, conducen a anemia como resultado de pérdidas sanguineas, inflamación, malabsorción o a consecuencia de las terapias farmacológicas. En algunas patologías como la cirrosis, Ell o neoplasias las causas son a menudo multifactoriales.

Esta revisión, pretende proporcionar un enfoque útil para la práctica clínica. Para ello se ha revisado la información actualizada acerca de la patogénesis, diagnóstico y tratamiento de la anemia vinculada a patologías digestivas y se han confeccionados cuadros y algoritmos para facilitar su comprensión.

Palabras clave: anemia, ferropenia, tracto gastrointestinal, hemorragia digestiva.

\section{ABSTRACT}

More and more patients diagnosed with anemia are referred to the gastroenterologist for evaluation. The need to carry out an adequate clinical approach and a correct interpretation of diagnostic tests has motivated this review.

Several digestive diseases frequently lead to anemia because of blood loss, inflammation, malabsorption, or drug therapies. In some of them such as cirrhosis, IBD or neoplasms, the etiology is multifactorial.

This review is intended to provide a useful approach to clinical practice. To this aim, updated information on the pathogenesis, diagnosis, and treatment of anemia related to digestive diseases has been reviewed, and tables and algorithms have been built to favor its understanding.

Keywords: anemia, iron deficiency, gastrointestinal tract, digestive bleeding

RESUMO

Cada vez mais pacientes diagnosticados com anemia são encaminhados ao gastroenterologista para avaliação. A necessidade de realizar uma abordagem clínica adequada e uma interpretação correta dos testes de diagnóstico motivou a revisão deste tema.

Vários distúrbios gastroenterológicos freqüentemente levam à anemia como resultado de perda de sangue, inflamação, má absorção ou pelas próprias terapias farmacológicas. Em algumas patologias como cirrose, DIl ou neoplasias, as causas costumam ser multifatoriais

Esta revisão visa fornecer uma abordagem útil à prática clínica Para esse fim, foram revisadas informações atualizadas sobre a patogênese, o diagnóstico e o tratamento da anemia associada à patologia digestiva e foram elaboradas tabelas e algoritmos para facilitar seu entendimento.

\section{IMPORTANCIA DEL TEMA Y OBJETIVOS}

La anemia es un problema de salud a nivel mundial. Un estudio de "carga de enfermedad" realizado en 2013 reporta que afecta a 27\% de la población mundial (1,93 billones de personas). Ochenta y nueve por ciento de esa carga se debe a los paises en desarrollo. En América Latina y el Caribe, un informe de OPS de 2016 reporta que la prevalencia de anemia en mujeres no embarazadas, entre 15 y 49 años, es de $18,7 \%$. Sin embargo, este promedio global no refleja las diferencias entre paises, que varian por ejemplo entre $1,5 \%$ en Nicaragua (2008) y 49,3\% en Haití (2012) (1). No se han encontrado datos de anemia en adultos en Uruguay en los últimos 5 años. La anemia puede ser la forma de presentación de múltiples patologías digestivas o la consecuencia de sus tratamientos, por lo que es causa frecuente de consulta al médico general y al gastroenterólogo.

Esta revisión, pretende proporcionar un enfoque útil para la práctica clínica. Para ello se ha revisado la información actualizada acerca de la patogénesis, diagnóstico y tratamiento de la anemia vinculada a patologias digestivas y se han confeccionados cuadros y algoritmos para facilitar su comprensión.

\section{DEFINICIONES Y CLASIFICACIONES}

La OMS define anemia como $\mathrm{Hb}$ menor a $13 \mathrm{mg} / \mathrm{dl}$ en varones, $12 \mathrm{mg} / \mathrm{dl}$ en mujeres no embarazadas y $11 \mathrm{mg} / \mathrm{dl}$ en mujeres embarazadas.

El hemograma completo con lámina permite valorar si se trata de anemia pura, mediante la exclusión del compromiso de la serie blanca y plaquetaria. Su severidad se evalúa a través de la repercusión hematimetrica y hemodinámica(2). Primariamente, la anemia leve se define por concentración de $\mathrm{Hb}$ mayor a $10 \mathrm{~g} / \mathrm{dl}$, moderada entre 10 y $8 \mathrm{~g} / \mathrm{dl}$ y severa cuando es menor de $8 \mathrm{~g} / \mathrm{dl}^{(3)}$. Según el contexto clínico del paciente, una anemia moderada del punto de vista hematimétrico puede transformarse en severa por su

Clinica de Gastroenterologia "Prof. Dra. Carolina Olano". Facultad de Medicina, Universidad de la República. Montevideo, Uruguay. Correo electrónico: digo.castaldelli@gmail.com. Correspondencia: Hospital de Clinicas. Av. Italia s/n (CP 11600). Montevideo, Uruguay Tel: +59824808472 
repercusión hemodinámica.

Se puede clasificar además según patogénesis, morfología de los glóbulos rojos y presentación clínica.

Los mecanismos patogénicos implicados son: la producción inadecuada y la pérdida de eritrocitos como resultado de hemorragia o hemólisis. Según estos mecanismos, la anemia se puede dividir en dos tipos: hiporregenerativa por función deteriorada de la médula ósea o regenerativa cuando la médula ósea responde adecuadamente a una masa baja de eritrocitos aumentando su producción ${ }^{(4)}$. Desde el punto de vista clínico se puede clasificar como aguda (generalmente sangrado o hemólisis) o crónica (vinculada a todas las demás afecciones).

La clasificación basada en parámetros básicos de la morfología de los glóbulos rojos, como el volumen corpuscular medio (VCM), clasifica la anemia en microcitica (menor a 80fl), normocitica (80-100fl) o macrocítica (mayor a 100fl), y permite un enfoque práctico. (Tabla 1).

Tabla 1. Diagnósticos diferenciales de la anemia

\begin{tabular}{|c|c|c|}
\hline Anemia microcitica & Anemia macrocitica & Anemia normocitica \\
\hline 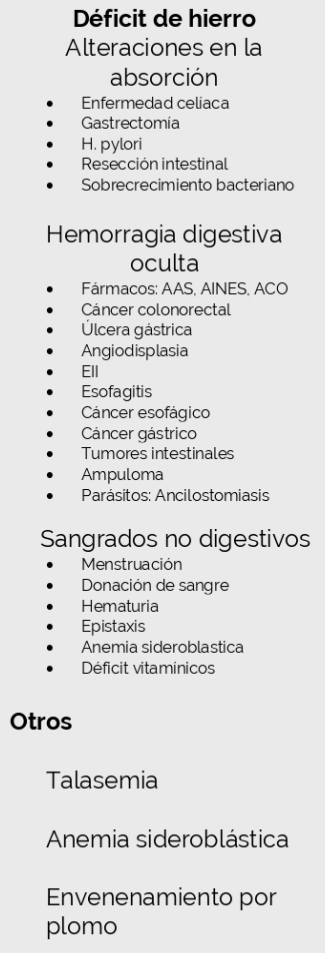 & 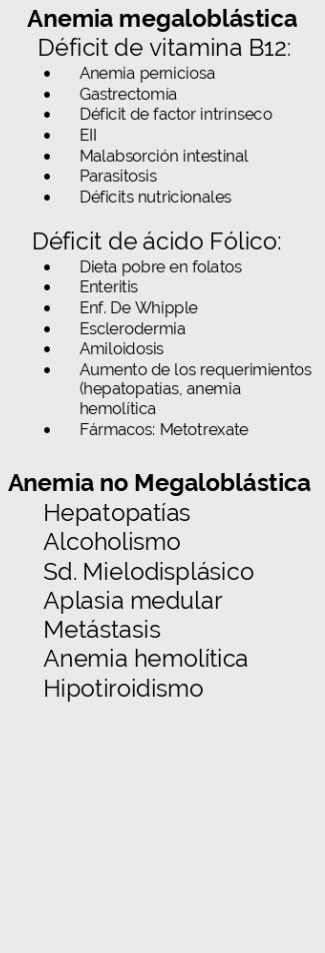 & $\begin{array}{l}\text { Enfermedades crónicas } \\
\text { Hepatopatías } \\
\text { Insuficiencia renal } \\
\text { Neoplasias } \\
\text { Déficits nutricionales } \\
\text { Déficit de hierro } \\
\text { Fármacos } \\
\text { Alcoholismo } \\
\text { Cirugías } \\
\text { Mielodisplásias }\end{array}$ \\
\hline
\end{tabular}

Fuente: elaboración propia.

La causa más frecuente de anemia microcitica es la AF. Otras incluyen talasemia y anemia sideroblástica. En la anemia normocítica la causa más prevalente es la anemia de la enfermedad crónica. Otras incluyen: anemia aplásica, hemólisis, poshemorrágica o condiciones que producen microcitosis y macrocitosis simultáneamente (por ejemplo, Enfermedad celiaca con afectación extensa). La prevalencia de anemia macrocitica es 1.7-3.9\%.
Las causas más frecuentes de anemia macrocitica incluyen deficiencia de vit B 12 y ácido fólico (anemia megaloblástica). Otras causas no megaloblásticas incluyen hipotiroidismo, fármacos (azatioprina, quimioterapia), síndromes mielodisplásicos, etc. Resulta interesante que más de $60 \%$ de los pacientes con macrocitosis no tienen anemia, evidenciando que la macrocitosis puede ser fisiológica en algunas circunstancias (lactantes, embarazo, herencia familiar) (5)

Del punto de vista etiológico la causa más frecuente de anemia es la deficiencia de hierro ( $\geq 60 \%$ ). Ésta ocurre en $2-5 \%$ de los hombres adultos y mujeres posmenopáusicas en los paises desarrollados y representa un motivo frecuente de derivación al gastroenterólogo. Puede deberse a ingesta 0 absorción reducida de hierro o pérdida de sangre. La ingesta reducida se ve fundamentalmente en dietas muy insuficientes o restrictivas.

Aproximadamente $2 / 3$ de los pacientes con AF presentan lesiones gastrointestinales ${ }^{(6)}$. La deficiencia de absorción de hierro es una causa menos frecuente. La absorción de hierro es un proceso complejo que ocurre principalmente en el duodeno. Varios factores determinan la eficacia de su absorción y una serie de condiciones médicas pueden interferir en ella. Los más importantes clínicamente son los trastornos que afectan las células de la mucosa responsables de la absorción de hierro (Enfermedad celíaca, por ejemplo), gastritis atrófica, infección por Helicobacter pylori y cirugia bariátrica. Los trastornos hereditarios que interfieren con la absorción de hierro son muy raros.

Otras etiologías de la anemia se deben a deficiencias por vit B12 y ácido fólico, éstas son menos frecuentes que la deficiencia de hierro y serán abordados más adelante.

La anemia inflamatoria o de la enfermedad crónica inicialmente se pensó que estaba asociada únicamente con enfermedades infecciosas, inflamatorias 0 neoplásicas. Sin embargo, más recientemente se la ha vinculado a otras como obesidad, diabetes mellitus, insuficiencia cardiaca congestiva, trauma severo y otras formas de activación inmune aguda o crónica(7).

La anemia es un sindrome, no una enfermedad per se, y por lo tanto conocer las posibles etiologías es esencial para dirigir los procedimientos diagnósticos y terapéuticos.

\section{ANEMIA FERROPÉNICA}

La deficiencia de hierro se define como la disminución del contenido total de hierro del organismo. La AF ocurre cuando la deficiencia de hierro es suficientemente severa para reducir la eritropoyesis ${ }^{(8)}$. 
Desde el punto de vista paraclínico la ferropenia se asocia a niveles descendidos de saturación de transferrina, sideremia y ferritina. Esta última es la prueba no invasiva más especifica para su diagnóstico. Según las guias de la Sociedad Americana de Gastroenterología 2020, niveles menores de 45 ng/ $\mathrm{ml}$ se consideran patognomónicos (sensibilidad de $85 \%$ y especificidad de $92 \%)^{(9)}$. Es importante tener en cuenta que la ferritina es un reactante de fase aguda, y su sintesis puede verse aumentada por los efectos de algunas citoquinas, como la IL1, neoplasias, infecciones o inflamación. Recientemente, ha sido reconocida la importancia del receptor soluble de la transferrina para diferenciar la AF de la anemia de la enfermedad crónica. Este receptor está únicamente aumentado en la $A F^{(8,9)}$. Otra forma de diferenciarlas sería titulando la hepcidina, principal regulador del metabolismo del hierro, ya que su producción esta disminuida en la AF y elevada si hay un proceso inflamatorio.

\section{METABOLISMO DEL HIERRO}

El hierro corporal total es de alrededor de $3500 \mathrm{mg}$, $65 \%$ se distribuye en los glóbulos rojos. En condiciones normales se utilizan $25 \mathrm{mg}$ de hierro diarios para la eritropoyesis ${ }^{(10)}$. La mayor fuente de hierro es proporcionada por los macrófagos que reciclan el hierro procedente de la destrucción de los eritrocitos.

A diario se consume alrededor de $10 \mathrm{mg}$ de hierro proveniente de la dieta. De éstos, solo 10\% es absorbido en los enterocitos ( 1 - 2 mg dia). El hierro proveniente de la dieta se encuentra mayoritariamente en forma férrica por lo que debe ser reducido a hierro ferroso para ser absorbido. De este modo, podrá atravesar el borde en cepillo de los enterocitos a través del transportador especifico (DMT1), que transporta metales divalentes como el hierro, el cobalto, el magnesio o el zinc ${ }^{(11)}$. La transferrina, una glucoproteina de 79,6KDa, es la responsable de su transporte en el plasma. El exceso de hierro se almacena en una glucoproteina llamada ferritina, compuesta por 24 subunidades unidas entre si por enlaces no covalentes, de forma esferoidal. Cuando el hierro es necesario para la eritropoyesis, es transferido a través de la membrana basolateral del enterocito mediante la ferroportina y entregado a los eritroblastos a través del receptor de la transferrina. En situaciones de ferropenia se produce una disminución de la sintesis de hemoglobina y aumento del número de mitosis de los eritroblastos que conducen a la microcitosis e hipocromia ${ }^{(12)}$

El regulador más importante de la homeostasis de hierro a nivel sistémico es la hepcidina. Se sintetiza en el hígado, actúa produciendo la internalización y degradación de la ferroportina, y en consecuencia inhibiendo el paso del hierro desde los enterocitos, macrófagos y hepatocitos a la sangre. Su sintesis se ve aumentada en respuesta a la sobrecarga de hierro y a la inflamación, y disminuida en respuesta a las situaciones de deficiencia de hierro, hipoxia o eritropoyesis ineficaz. Las situaciones de inflamación aumentan los niveles de hepcidina, a través de señales mediadas por interleuquinas, contribuyendo asi a la anemia asociada a los procesos crónicos. Por el contrario, en el caso de anemia ferropénica, la hepcidina se encuentra disminuida para lograr el aumento de la absorción de hierro a nivel intestinal y liberación desde los sitios de depósito(13) (Figura 1).

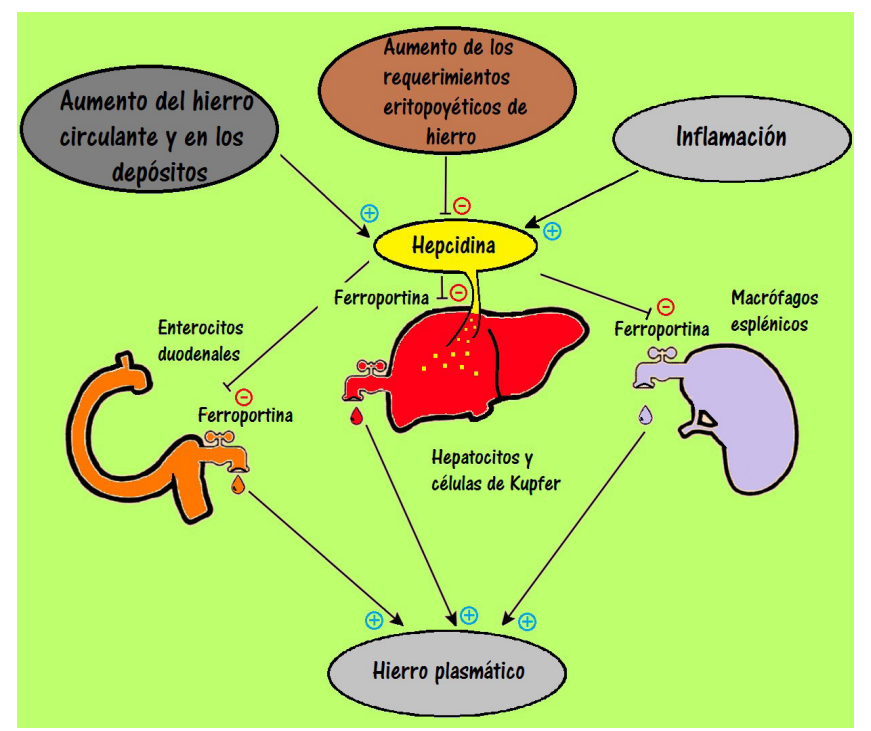

Figura 1. Rol de la hepcidina en la homeostasis del hierro La hepcidina actúa produciendo la degradación de la ferroportina inhibiendo el paso del hierro desde los enterocitos, macrófagos y hepatocitos a la sangre. Su sintesis se ve aumentada en respuesta a la sobrecarga de hierro y a la inflamación, y disminuida en situaciones de aumento de los requerimientos eritropoyéticos de hierro. Al disminuir la hepcidina se incrementa la absorción intestinal de hierro y su liberación desde los depósitos.

Fuente: elaboración propia.

\section{HISTORIA CLÍNICA Y EXPLORACIÓN FÍSICA}

La anemia causa hipoxia tisular y desencadena mecanismos de compensación. Como consecuencia se producen sintomas y signos característicos del sindrome funcional anémico como: palidez cutáneomucosa, fatiga, mareos, disnea, acúfenos, fosfenos, somnolencia, taquicardia, soplos y dolor anginoso(6).

Importa determinar si se trata de anemia pura descartando el compromiso de otras series (sangrados cutáneos, mucosos o viscerales e infecciones), asi como buscar la presencia de adenopatías y visceromegalias.

Específicamente la anemia ferropénica se caracteriza por depapilación de los bordes de la lengua, coiloniquia, pelo quebradizo, trastornos de la conducta alimentaria como hábito de pica, síndrome de piernas inquietas y en casos severos, disfagia por la presencia de membranas esofágicas (Sindrome de Plummer 
Vinson)(1).

Es necesario pesquisar sintomas de la esfera digestiva que orienten a la etiologia: dolor abdominal, pirosis, regurgitaciones, diarrea, distensión abdominal, constipación o alteración del tránsito digestivo. Es importante conocer si el paciente tiene una dieta baja en hierro, si recibe anticoagulantes o AINE. Los antecedentes personales de enfermedades autoinmunes, insuficiencia renal, enfermedades hematológicas o resecciones quirúrgicas pueden orientar al diagnóstico etiológico. Del punto de vista gineco-obstétrico es necesario descartar la presencia de menometrorragias, hipermenorrea, metrorragia postmenospáusica, periodos intergenésico muy cortos que justifiquen las pérdidas, así como la presencia de menarca tardia y menopausia precoz que orienten a EC. Los antecedentes familiares de Ell, neoplasias del tracto gastrointestinal, EC, talasemia, Rendu Osler Weber, entre otros, cobran relevancia.

\section{ENFOQUE DIAGNÓSTICO}

El enfoque diagnóstico de la AF es diferente en mujeres premenopaúsicas de los hombres y mujeres postmenopáusicas por lo que se tratarán por separado (Figura 2).

\section{ESTUDIO DE ANEMIA FERROPÉNICA EN MUJERES PREMENOPÁUSICAS}

La AF se produce en 5-12\% de mujeres premenopáusicas. Por frecuencia, las principales etiologías son los sangrados ginecológicos, la deficiencia nutricional o el incremento de la demanda durante el embarazo y la lactancia. Las causas gastrointestinales son infrecuentes (13-30\%) y son debidas a malabsorción de hierro o pérdida de sangre por el tracto gastrointestinal(7). Sus etiologias más frecuentes están en los sectores proximales: EC, gastropatía por AINE, gastritis atrófica e infección por H. pylori. Los mecanismos de ferropenia vinculados a la infección por H. pylori son: disminución de la absorción (menor captación del hierro de la dieta, reducción de ácido ascórbico, inflamación de la mucosa que eleva la hepcidina) y aumento de las pérdidas (microerosiones).

Otras causas menos frecuentes de AF en estas pacientes incluyen úlcera péptica, cáncer gástrico, parasitosis (giardiasis, por ejemplo), sobrecrecimiento bacteriano, gastrectomía parcial o total, resección o bypass intestinal(7). Entre las lesiones colónicas se destaca la Ell, especialmente Enfermedad de Crohn, siendo infrecuente el cáncer colorrectal en este grupo etario.

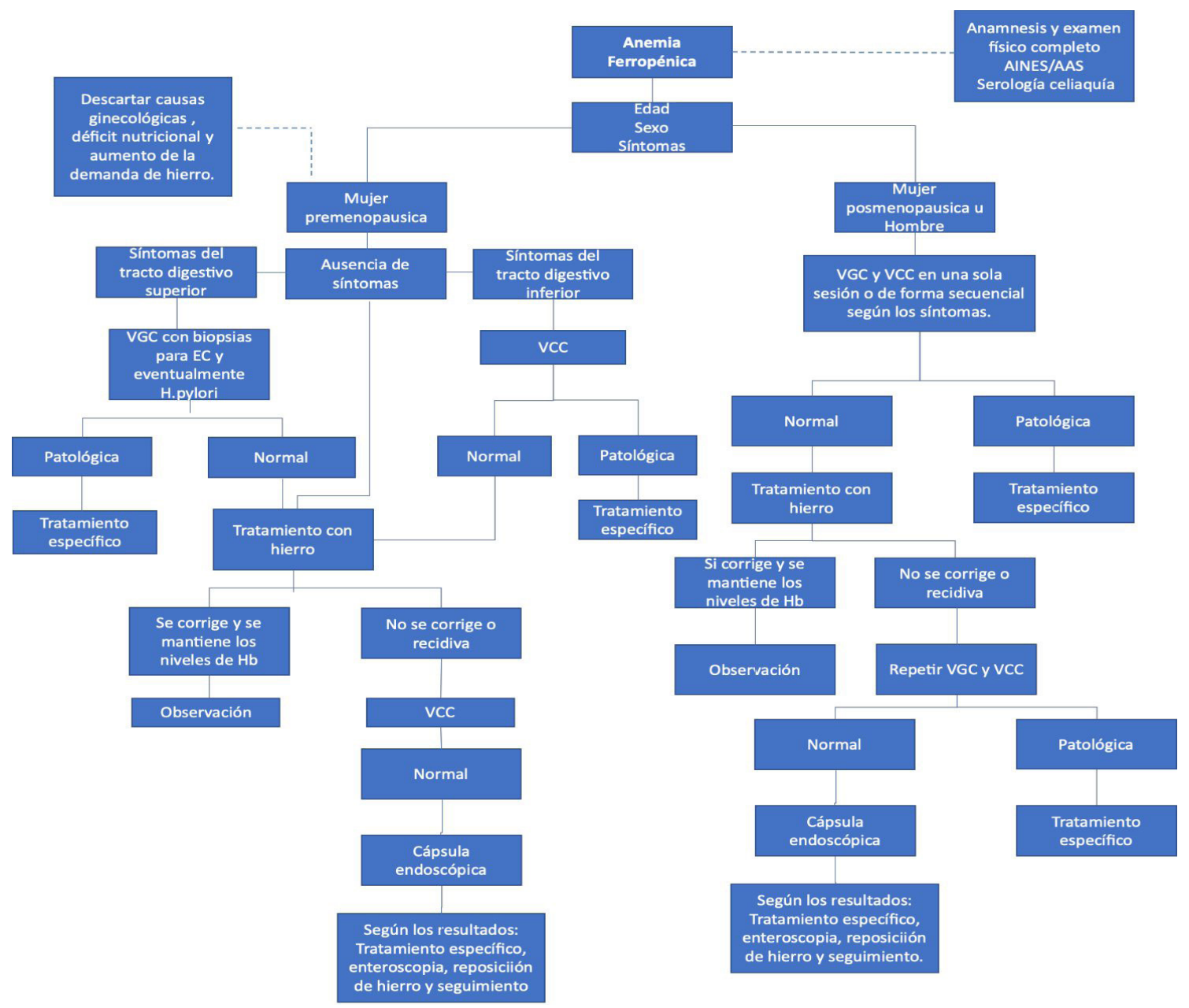

Figura 2. Algoritmo para el diagnóstico etiológico de la anemia ferropénica

Fuente: elaboración propia. 
El rendimiento diagnóstico de las diferentes exploraciones no ha sido adecuadamente evaluado y no existe consenso sobre qué exploraciones realizar y en qué orden deben solicitarse. Las guías clinicas recomiendan realizar al menos hemograma, metabolismo del hierro y serología para EC (anticuerpos anti-transglutaminasa y anti-antiendomisio con dosificación de $\lg A)^{(14)}$. Se considerará realizar VGC en caso de presentar factores de riesgo adicionales: edad $>40$ años, hemoglobina $<10 \mathrm{~g} / \mathrm{dl}$, melenas, dolor abdominal, pérdida de peso o falta de respuesta a la suplementación con hierro(15). Es mandatorio la exploración hasta duodeno distal y la toma de biopsias gástricas para investigación de la infección por H. pylori y duodenales (bulbo y DIl para descartar atrofia vellositaria) (Figura 3). La VCC debe reservarse a pacientes con sintomas colónicos, antecedentes familiares de primer grado de cáncer colorrectal o con AF persistente después de la suplementación de hierro y corrección de las posibles pérdidas. Es esencial que tenga una adecuada preparación y visualización de la última asa ileal.

Si todos los estudios anteriores son negativos se deberá estudiar el intestino delgado como se especifica más adelante.

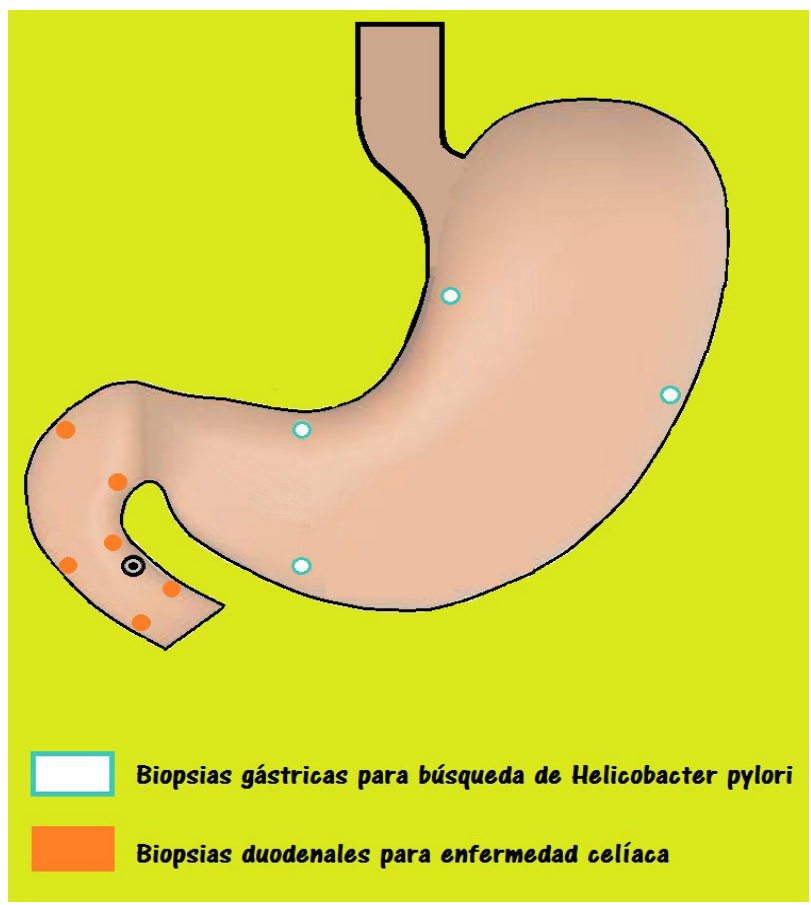

Figura 3. Toma de biopsias en la VGC por anemia ferropénica. Se deben realizar 2 biopsias de bulbo duodenal y 4 biopsias de duodeno II (2 proximales y 2 distales a la papila) en frascos separados para valoración de EC. Para búsqueda de H. pylori, según Mastrich V, se deben realizar 2 biopsias de cuerpo alto en la curvatura menor y mayor y 2 biopsias de antro en curvatura menor y mayor.

Fuente: elaboración propia

\section{ESTUDIO DE ANEMIA FERROPÉNICA EN MUJERES POSMENOPÁUSICAS Y HOMBRES}

En este grupo la causa más frecuente de AF es la pérdida crónica a través del tubo digestivo y la malabsorción de hierro de la dieta (EC, gastrectomizados, gastritis atrófica).

La evaluación inicial se realiza con hemograma, metabolismo del hierro y serología especifica para EC. Comenzar la exploración endoscópica con VGC - VCC se hará de acuerdo con los síntomas o en una misma sesión endoscópica para acelerar el proceso diagnóstico y optimizar el acto anestésico. La combinación de VGC y VCC permite identificar el origen de la AF en 60 a $70 \%$ de los $\operatorname{casos}^{(16)}$. De igual manera que en las mujeres premenopáusicas, los estudios endoscópicos deben optimizarse.

En caso de no obtener el diagnóstico mediante el estudio endoscópico alto y bajo y descartada la EC con marcadores serológicos y biopsia, se convierte en un desafio clínico que se denomina "sospecha de sangrado de intestino delgado" en su forma oculta. Una alternativa sería repetir las exploraciones previas lo que podría identificar hasta 10\% de lesiones adicionales en el tracto gastrointestinal superior, y hasta $7 \%$ en el colon $^{(17)}$. Las lesiones más frecuentemente inadvertidas son las de origen vascular ${ }^{(18)}$

Una estrategia aceptada en estos casos sería corregir las deficiencias dietéticas, suspender (si es posible) el tratamiento con AINE o antiagregantes, realizar una pauta de tratamiento con hierro y controlar los niveles de hemoglobina y ferritina. Si el paciente presenta una respuesta adecuada, sin recidiva de la AF, parece seguro no realizar nuevas exploraciones. En caso de una respuesta inadecuada o rápida recidiva de la AF deberá plantearse el estudio del intestino delgado.

La CE es la primera línea de exploración, la enteroscopia quedaría reservada para tratar lesiones detectadas por la CE, cuando ésta es negativa o en anemia persistente a pesar del tratamiento.

Existen pocos trabajos que hayan evaluado específicamente el rendimiento diagnóstico de la CE en AF. Una revisión sistemática presentada en 2012 y en base únicamente a 3 estudios mostró un rendimiento diagnóstico de $66 \%$. Un trabajo posterior realizado en Uruguay arrojó un rendimiento de 50\% en un grupo altamente seleccionado de pacientes con AF y donde se consideraron únicamente lesiones endoscópicamente relevantes ${ }^{(19)}$.

La CE tiene la ventaja de no ser invasiva y la desventaja de no ser terapéutica. Permite sin embargo guiar el acceso anterógrado o retrogrado de la enteroscopía profunda en caso de necesidad de biopsias o terapéutica (gas argón, polipectomía, dilatación, etc). Saurin y colaboradores idearon una clasificación para determinar mediante CE que lesiones tiene alto, incierto o ningún potencial de sangrado. Las lesiones con alto potencial de sangrado son: angioectasias (29$40 \%$ ), erosiones y úlceras ( $7 \%$ ); menos frecuentemente 
tumores, y varices intestinales ${ }^{(20)}$.

La enteroscopia de pulsión permite identificar el origen de las pérdidas hemáticas en 30-50\% de los pacientes con VGCyVCC normal. Elúnico inconveniente es que explora no más allá de $70 \mathrm{~cm}$ del ángulo de Treitz. La enteroscopía profunda (mono balón, doble balón o espiral) permite la exploración de la totalidad del intestino delgado en una o más sesiones.

Un estudio retrospectivo observó que el tratamiento de las lesiones detectadas durante la enteroscopia se asociaba a la ausencia de recidiva de la AF en $71 \%$ y aquellos con enteroscopia normal no presentaban reaparición de la $A F$, por lo que se aconsejaba no realizar nuevas exploraciones ${ }^{(21)}$

La entero TC y la entero RMN permiten visualizar la totalidad de la pared intestinal y no solo la superficie de la mucosa, por lo que resultan útiles en el caso de sospecha de Ell o para detectar tumores del intestino delgado. Sin embargo, la mayor parte de las lesiones del intestino delgado que causan AF son lesiones planas, no detectables mediante las exploraciones radiológicas.

De acuerdo con la última terminología proporcionada por el Colegio Americano de Gastroenterología de 2015, en el caso de los pacientes en los que no se ha encontrado etiología por VGC, VCC, CE o estudios de imagen se considera que se trata de una hemorragia digestiva de origen oscuro y oculto.

\section{TRATAMIENTO}

El abordaje terapéutico de la AF debe basarse en el tratamiento de la causa desencadenante y la adecuada reposición. Independiente de la causa, deberán administrarse suplementos de hierro para corregir las cifras de $\mathrm{Hb}$, los indices eritrocitarios y normalizar los depósitos corporales. La elección de la vía de reposición depende de la clínica del paciente, así como de los valores de $\mathrm{Hb}$. Se prefiere utilizar hierro oral si la $\mathrm{Hb}$ es mayor a $10 \mathrm{~g} / \mathrm{dl}$ e I/ $\mathrm{V}$ si es menor.

Para calcular el déficit de hierro se utiliza la fórmula de Ganzoni:

déficit de hierro $(\mathrm{mg})=$ Peso corporal $(\mathrm{kg}) \times\left(\mathrm{Hb}\right.$ objetivo $-\mathrm{Hb}$ real) $(\mathrm{g} / \mathrm{dll}) \times 2.4^{4}$ ferritina

\section{SUPLEMENTOS DE HIERRO ORAL}

Las sales ferrosas se absorben mejor, disponen de más hierro elemental y son más económicas que las sales férricas, por ello se han convertido en el tratamiento de elección.

Se recomiendan la dosis de 2-3 mg de hierro elemental/kg peso al día (100 a $200 \mathrm{mg}$ de hierro dia). Un tratamiento adecuado incrementará las cifras de $\mathrm{Hb}$ alrededor de $2 \mathrm{~g} / \mathrm{dl}$ a las 3-4 semanas La suplementación de hierro debe continuarse 3 meses tras la normalización de las cifras de Hb para llenar depósitos ${ }^{(22)}$.

La administración conjunta con 250 a 500 mg de ácido ascórbico mejora la absorción de hierro, pero puede aumentar la intolerancia gástrica. Se desaconseja la administración rutinaria de hierro asociado a otras sustancias (por ejemplo, B12, polivitamínicos) ya que la cantidad de hierro elemental puede ser insuficiente.

La respuesta al tratamiento se evidencia mediante el aumento de los reticulocitos a los 7-10 días.

La adherencia al tratamiento con hierro oral es relativamente baja debido a efectos adversos hasta en $20 \%$. El efecto adverso más frecuente es la intolerancia digestiva en forma de náuseas, dolor abdominal, diarrea o estreñimiento. En estos casos puede indicarse dosis menores o su administración con las comidas, ya que mejoran la tolerancia y, por lo tanto, la adherencia, aunque implican un tratamiento más prolongado por disminución de su absorción ${ }^{(23)}$.

\section{SUPLEMENTOS DE HIERRO ORAL}

Es la vía de administración reservada para casos de intolerancia o refractariedad al hierro oral.

Existen distintos preparados de hierro endovenoso que tienen en común una estructura compuesta por un núcleo central polinuclear de hierro férrico elemental rodeado por una cubierta de carbohidratos que actúa como estabilizador previniendo la liberación incontrolada de hierro libre tóxico ${ }^{(24)}$.

El hierro sacarosa es administrado en dosis de 200 mg en una infusión de 30 min, 3 veces por semana. Es un fármaco bien tolerado, con una tasa de efectos adversos muy baja (6 de cada 10 millones de dosis).

La carboximaltosa férrica tiene la ventaja de presentar un tiempo de infusión muy corto (15 min) y permitir la administración de hasta 1000 mg por semana (Tabla 2).

La respuesta al tratamiento es más rápida que con el sulfato ferroso oral. En pacientes con Ell, la carboximaltosa fue superior al tratamiento oral con preparados de sulfato ferroso, con mayor tasa de respuesta, mayor rapidez en la recuperación de la anemia, mejor tolerancia y mayor calidad de vida. Asimismo, se ha demostrado su superioridad frente a los preparados orales en pacientes con insuficiencia renal crónica ${ }^{(25)}$. La principal desventaja de este preparado es su mayor costo. Un efecto adverso reportado con relativa frecuencia es el dolor óseo, causado por la rápida expansión de la masa eritrocitaria en la medula ósea secundaria a la carga de hierro.

El hierro intravenoso permite una rápida respuesta inicial de las cifras de $\mathrm{Hb}$, pero a las 12 semanas es similar a la observada con el tratamiento con hierro oral. 
Tabla 2. Características de las dos principales formulaciones de hierro i/v utilizadas

\begin{tabular}{|c|c|c|}
\hline Caracteristicas & Hierro Sacarosa & Hierro Carboximaltosa \\
\hline Estabilidad & Baja-moderada & Alta \\
\hline Reacciones infusionales & $\begin{array}{l}\text { Poco frecuente } \\
\text { (1:20000dosis) }\end{array}$ & Rara \\
\hline Dosis máxima & 200mg Fe & 1000mg Fe elemental \\
\hline Dosis semanal & $600 m g$ Fe elemental & $1000 \mathrm{mg}$ Fe elemental \\
\hline Rapidez de infusión & 30 minutos & 15 minutos \\
\hline Dosis prueba & No requiere & No requiere \\
\hline Costo & Bajo & Elevado \\
\hline $\begin{array}{l}\mathrm{N}^{\circ} \text { de visitas al hospital para } \\
\text { administrar 1000mg de } \mathrm{Fe}\end{array}$ & 5 & 1 \\
\hline
\end{tabular}

Fuente: elaboración propia.

\section{TRANSFUSIÓN SANGUÍNEA}

La transfusión sanguínea se realizará con el objetivo de conseguir niveles seguros de $\mathrm{Hb}$ (no niveles normales). Aunque cada concentrado de glóbulos rojos aporta aproximadamente $200 \mathrm{mg}$ de hierro, no debe ser utilizada como terapia de reposición. Debe reservarse para pacientes con anemia sintomática, especialmente en los casos con alto riesgo de descompensación cardiovascular. El nivel de $\mathrm{Hb}$ no debe ser el único factor para decidir su utilización. Esta decisión debe ser basada además en la necesidad de mejorar los signos y síntomas clínicos y prevenir la morbilidad y mortalidad en estos pacientes. Las pautas de transfusión recomiendan la administración de los concentrados globulares de uno en uno hasta la estabilización ${ }^{(26)}$

\section{SEGUIMIENTO}

La guía británica para manejo de la AF propone controles trimestrales durante un año, posteriormente al año y en adelante si reaparecen los sintomas. En caso de recidiva deberá revalorarse y considerar estudios adicionales ${ }^{(14)}$.

\section{ANEMIA POR DÉFICIT DE VITAMINA B12 Y ÁCIDO FÓLICO}

La anemia megaloblástica se debe con mayor frecuencia a la deficiencia especifica de la vit B12 (cobalamina) y ácido fólico, que son necesarios para la sintesis de $\mathrm{ADN}^{(5)}$.

La vit B12 es sintetizada por el ser humano en cantidades mínimas. En el estómago la vit B12, se separa de las proteinas de los alimentos gracias al pH ácido y a las enzimas pancreáticas. Se absorbe por medio de dos mecanismos; uno pasivo (absorbe menos de $1 \%$ de la dosis oral y ocurre en la mucosa bucal, duodeno y en el íleon) y otro activo (que permite la absorción de más del $90 \%$ de la dosis oral). Este último mecanismo se lleva a cabo en el íleon distal y depende del factor intrinseco gástrico. Éste es secretado por las células parietales del estómago, se une a la vit B12 y forman un complejo que es reconocido por receptores presentes en las células de la mucosa ileal. Los depósitos de vit B12 se localizan en el hígado, son muy abundantes (2-5 mg), lo que hace que su deficiencia tarde años en manifestarse ${ }^{(27)}$.

La deficiencia de vit B12 está causada por disminución en la ingesta (vegetarianos estrictos) o alteraciones en su absorción y transporte como en: enfermedades gástricas (anemia perniciosa, gastrectomía, gastritis crónica atrófica), enfermedades del intestino delgado (malabsorción, resección ileal, sindrome de asa ciega), insuficiencia pancreática y fármacos (IBP, anti $\mathrm{H}_{2}$, metformina, colchicina, neomicina, colestiramina).

La anemia perniciosa es la principal causa de deficiencia de vit B12. Es una enfermedad autoinmune debida a la presencia de anticuerpos dirigidos contra las células parietales y/o contra el factor intrínseco, lo que produce gastritis crónica atrófica (autoinmune o tipo A) en el cuerpo y fundus del estómago. No suele presentar sintomas y produce aclorhidria. Se asocia a otras enfermedades autoinmunes (tiroiditis, diabetes mellitus tipo I, vitiligo, etc.)(28)

Otra causa de anemia megaloblástica es la deficiencia de ácido fólico. Las necesidades diarias del adulto varian entre 50 a $100 \mu \mathrm{g}$. El ácido fólico se absorbe principalmente en duodeno y yeyuno proximal. El cuerpo almacena alrededor de $5 \mathrm{mg}$ de ácido fólico en el hígado, suficiente para cubrir las necesidades fisiológicas durante 3 a 4 meses. La deficiencia de ácido fólico puede estar relacionada con la disminución de la ingesta (alcoholismo o desnutrición) o aumento de la demanda (embarazo, malabsorción, hemólisis y hemodiálisis) ${ }^{(29)}$.

\section{HISTORIA CLÍNICA Y EXPLORACIÓN FÍSICA}

Por lo general, la anemia se desarrolla gradualmente y los sintomas están presentes solo en pacientes con anemia grave. Los sintomas y signos comunes incluyen SFA, depapilación de la lengua, glositis (que en este caso recibe el nombre "de Hunter") y esplenomegalia. De presentarse ictericia puede deberse a hemólisis.

Las manifestaciones clínicas predominantes de la deficiencia de vit B12 son neurológicas: parestesias y sindrome cordonal posterior (alteración de la sensibilidad vibratoria, ataxia, hiper o hiporreflexia). Pueden presentar dolores lancinantes causados por neuropatía periférica que afecta principalmente a las extremidades inferiores. Con menos frecuencia, puede haber trastornos visuales causados por atrofia óptica. Otras manifestaciones incluyen: alteraciones de la memoria o de la concentración, demencia, depresión, angustia y estrés ${ }^{(27,28)}$

El examen físico generalmente muestra pérdida de la sensibilidad vibratoria y propiocepción con una prueba 
de Romberg positiva. También se presenta, el reflejo de Babinski, la hiporreflexia y clonus. Estos trastornos neurológicos pueden no ser reversibles después de la terapia de reemplazo.

\section{ENFOQUE DIAGNÓSTICO}

El diagnóstico de la anemia megaloblástica debe sospecharse frente a la presencia de los sintomas antes mencionados y/o los resultados del hemograma, donde se observa anemia con macrocitosis. El recuento de reticulocitos es bajo. En el frotis de sangre periférica los megaloblastos, aunque no son patognómicos, son altamente sugestivos de anemia megaloblástica. Otros cambios que se pueden observar son los cuerpos de Howell-Jolly, anisocitosis y poiquilocitosis, además pueden verse neutrófilos hipersegmentados. Para el diagnóstico es imprescindible evaluar los niveles plasmáticos y séricos de vit B12 y ácido fólico. La deficiencia de vit B12 es considerada cuando los niveles están por debajo de $200 \mathrm{pg} / \mathrm{ml}$. Se solicitan simultáneamente los valores de ácido fólico y hierro ya que puede asociarse carencia de ambos. Asimismo, se debe solicitar los anticuerpos contra factor intrinseco (más especifico) y células parietales: positivos en $75 \%$ de los casos de anemia perniciosa, aunque su negatividad no excluye el diagnóstico(29).

Para el estudio etiológico, la VGC cobra relevancia ya que permite evidenciar elementos endoscópicos de atrofia gástrica: visualización de los vasos submucosos, disminución de la altura/extensión de los pliegues gástricos, aumento de la refractariedad de la luz. Se confirma histológicamente por la toma de biopsias del antro, sinus y cuerpo gástrico (Mapeo de Sydney)(30). Además la VGC permite valorar elementos de atrofia duodenal, como pliegues duodenales festoneados, patrón mucoso en mosaico, bulbo nodular, asi como disminución del número/altura de pliegues y visualización de los vasos submucosos. Se confirman con biopsias de bulbo y duodeno II ya descriptas previamente.

\section{TRATAMIENTO}

En casos de deficiencia de vit B12, el tratamiento se centra en inyecciones intramusculares de hidroxocobalamina. Por lo general, los pacientes reciben $1000 \mu \mathrm{g}$ de vit B12 diariamente en la primera semana de tratamiento, luego una dosis semanal durante el primer mes y posteriormente dosis mensuales hasta la corrección de los niveles. En algunos casos, en particular, en pacientes con anemia perniciosa, gastrectomía total previa o resección ileal extensa, el tratamiento con vit B12 es de por vida.

En el caso de la deficiencia de ácido fólico, es esencial determinar la ausencia de deficiencia concomitante de vit B12 antes de comenzar la terapia. De hecho, la neuropatía puede empeorar si se administran grandes dosis de ácido fólico en presencia de una deficiencia concomitante. El tratamiento con ácido fólico, de 1 a $5 \mathrm{mg} /$ dia, generalmente se administra por vía oral. En pacientes con malabsorción, se recomienda la preparación parenteral. Para evitar recaídas, el tratamiento debe continuar por un mínimo de dos años ${ }^{(31)}$

\section{ANEMIA EN PATOLOGÍAS GASTROENTEROLÓGICAS ESPECIFICAS}

\section{ENFERMEDAD CELÍACA}

Es una enfermedad inflamatoria de origen autoinmune que afecta la mucosa del intestino delgado en pacientes genéticamente susceptibles y cuyo desencadenante es la ingesta de gluten. Se presenta con gran heterogeneidad clínica y en todos los grupos etarios ${ }^{(32)}$.

La anemia podría ser la única expresión clínica de la enfermedad, y la AF se considera la manifestación clínica extraintestinal más frecuente. Tiene una patogénesis multifactorial $y$, aunque en la mayoría de los casos es consecuencia de deficiencia de hierro, puede ser causada por deficiencias de ácido fólico o vit B12, anemia de las enfermedades crónicas o ser consecuencia de otras enfermedades asociadas ${ }^{(33)}$.

Los celiacos que se presentan con anemia tienen una enfermedad más grave que los que se presentan con diarrea. Un estudio norteamericano, sugiere que la presentación con anemia se asoció con atrofia vellositaria severa y predijo mayor gravedad de la enfermedad; asimismo los pacientes con anemia tenían mayor prevalencia de osteoporosis y niveles séricos más altos de antitransglutaminasa ${ }^{(34)}$.

La AF es un hallazgo frecuente en pacientes con EC manifiesta (10-20\% de los casos) y se debe principalmente a la deficiencia de hierro como consecuencia de su malabsorción en el duodeno. En pacientes con AF y falta de respuesta al tratamiento con hierro oral se debe pensar en esta enfermedad. Un metaanálisis reciente encontró que más de $3 \%$ de los pacientes con anemia por deficiencia de hierro tenían evidencia histológica de EC, lo que refuerza la necesidad de su detección ${ }^{(27)}$.

Es de vital importancia incluir la biopsia duodenal en el protocolo de diagnóstico de AF. Asumir que la presencia de lesiones gastrointestinales menores justifica la AF soslayando la necesidad de biopsia duodenal, podría dejar sin diagnosticar una condición clínica importante y tratable ${ }^{(35)}$.

Por otro lado, varios estudios en pacientes celiacos 
adultos han demostrado un mayor riesgo de deficiencia de ácido fólico, que puede alcanzar hasta $20-30 \%$ al momento del diagnóstico. Antes de la absorción, el ácido fólico debe ser desconjugado por una peptidasa de membrana. El daño de la mucosa intestinal en la EC puede afectar la actividad enzimática que conduce a su deficiencia ${ }^{(36)}$

Un trabajo realizado en este servicio, no publicado, demostró que luego del inicio de la dieta libre de gluten, la anemia revirtió en $64 \%$ de un grupo de pacientes celíacos. Sin embargo, los valores de ácido fólico inicialmente normales tendieron al descenso en $70 \%$ de los pacientes. Es frecuente que no exista suplementación con ácido fólico de las harinas que utilizan los celiacos para elaboración de sus alimentos. Esto podria explicar la carencia a largo plazo en celíacos bajo dieta libre de gluten.

Autores como Garcia-Manzanares y Lucendo informaron una prevalencia de deficiencia de vit B12 entre $8 \%$ y $41 \%$ en pacientes con diagnóstico reciente de EC. Las causas podrian relacionarse con disminución de la acidez gástrica, consumo de cobalamina debido al hallazgo frecuente de sobrecrecimiento bacteriano, gastritis autoinmune y disminución de la eficiencia de factor intrínseco o incluso disfunción del intestino delgado distal(33, 37).

La prevalencia de anemia de enfermedades crónicas en la EC varia de 3.9-17\%(37). Se plantea como mecanismos patogénicos una producción defectuosa de eritropoyetina endógena, asi como la producción de citoquinas proinflamatorias que estimulan la producción de hepcidina ${ }^{(38)}$.

\section{ENFERMEDAD INFLAMATORIA INTESTINAL}

La anemia en la Ell es causa de aumento de los requerimientos transfusionales, así como de la admisión y estancia media hospitalaria(35).

Es mayor su frecuencia en la enfermedad de Crohn y en pacientes hospitalizados (70\%)(39). Las causas suelen ser multifactoriales, entre ellas la más importante es la ferropenia que se presenta hasta en $45 \%$ de estos pacientes. Es consecuencia del sangrado de la mucosa inflamada, absorción disminuida si el duodeno está afectado, restricciones dietéticas y la presencia de múltiples cirugías ${ }^{(40)}$.

La valoración de la deficiencia de hierro es importante en esta patología. Para ello es necesario tener en cuenta que en pacientes sin evidencia bioquímica y/o clínica de inflamación, para definir ferropenia es necesario valores de ferritina sérica inferiores a $45 \mathrm{ug} / \mathrm{l}$. Sin embargo, en presencia de inflamación, se considera ferropenia con valores inferiores a $100 \mathrm{ug} / \mathrm{l}^{(41)}$.

También es frecuente la anemia de los trastornos crónicos producida por disminución de la vida media y de la producción de los hematies en el contexto de inflamación crónica. (Ver metabolismo del hierro)(7).

La anemia por deficiencia de vit B12 y ácido fólico es menos frecuente, explicando solo $1.5 \%$ y es mayormente de origen carencial. La deficiencia de vit B12 se debe a malabsorción por afectación extensa de la mucosa, sobrecrecimiento bacteriano, afectación gástrica en la Enfermedad de Crohn y enteropatía pierde proteinas. La deficiencia de ácido fólico es consecuencia de la ingesta de fármacos mielo-tóxicos como la sulfasalazina y tiopurinas.

Debido al carácter multifactorial de la anemia, es necesario antes de instaurar un régimen terapéutico, intentar identificar su etiología. La primera maniobra terapéutica será el correcto control de la inflamación ${ }^{136}$. 42).

Las guias ECCO sobre anemia en Ell recomiendan realizar controles analíticos que incluyan hemograma, ferritina sérica y proteina $C$ reactiva cada 6-12 meses en sujetos en remisión y cada 3 meses en pacientes ambulatorios con enfermedad activa. Aquellos casos con riesgo de deficiencia de vit $\mathrm{B} 12$ o ácido fólico también deben ser específicamente controlados, midiendo sus niveles al menos anualmente. En sujetos con resección extensa del intestino delgado, Enfermedad de Crohn ileal amplia y portadores de reservorios el control debe ser más frecuente ${ }^{(43)}$.

La reaparición de la anemia es habitual (50\% a los 10 meses). La recurrencia es menor si se han normalizado los depósitos de ferritina tras el tratamiento. Una ferritina postratamiento $>400 \mathrm{ug} / \mathrm{l}$, se relaciona con tasas de recurrencia inferiores ${ }^{(44)}$.

El objetivo del tratamiento es normalizar la $\mathrm{Hb}$ y los depósitos de hierro lo que se verá reflejado en una mejoría de la calidad de vida. Actualmente se prefiere el hierro intravenoso frente a la vía oral, dado que la absorción intestinal de hierro puede estar comprometida por el ascenso de hepcidina. Además, evita sus efectos adversos gastrointestinales, es eficaz y rápido en casi todos los escenarios y asegura el cumplimiento. En general, la indicación principal es anemia con componente ferropénico «moderadagrave». También estará indicado en aquellos pacientes que, aún con cifras mayores, no toleren o no respondan al hierro oral y en aquellos en que se necesite una rápida recuperación de la anemia o se precise asociar eritropoyetina. El uso de transfusiones está restringido a casos refractarios o situaciones agudas que ponen en riesgo la vida ${ }^{(10)}$

Una vez corregida la anemia, se recomienda valorar ferropenia cada 3 meses durante el primer año, y cada 6-12 meses posteriormente. Tratar la enfermedad correctamente es clave para prevenir la reaparición de anemia(43). 


\section{HEMORRAGIA DIGESTIVA}

La hemorragia gastrointestinal aguda o crónica constituye un problema relevante cuya mortalidad oscila entre 3-14\% al año(45).

La incidencia de la HDA ha disminuido en las últimas décadas, debido en parte al tratamiento de la infección por $\mathrm{H}$. pylori y al uso de gastroprotección en pacientes que requieren AINE o antiagregantes plaquetarios. Por el contrario, la incidencia de HDB ha experimentado un crecimiento relacionado con el envejecimiento poblacional, la presencia de comorbilidades y el aumento de la necesidad de tratamientos anticoagulantes y antiagregantes.

El manejo agudo de la hemorragia digestiva está bien estandarizado y se basa en la evaluación de la magnitud del sangrado y medidas de reanimación inmediata, así como estratificación del riesgo basado en las caracteristicas del paciente (edad, comorbilidades), y del propio sangrado (etiología, cuantía y/o velocidad de la extravasación, y estigmas endoscópicos premonitorios de recidiva)(46).

Tras una hemorragia digestiva aguda la pérdida de masa eritrocitaria y la necesidad de regeneración de hematies contribuyen al desarrollo de una deficiencia de hierro que debe ser corregida para evitar el desarrollo de AF. Todavía faltan pautas claras para el manejo de estos pacientes. Un estudio retrospectivo evidenció que más de $80 \%$ de los pacientes ingresados en el hospital por HDA no variceal presentaban anemia al momento del alta ${ }^{(47)}$. De éstos, solo $16 \%$ recibió una recomendación para comenzar tratamiento con suplementos de hierro por vía oral, lo que demuestra que la anemia posterior al episodio agudo no es tenida en cuenta en la mayoria de los casos.

Se ha evaluado el impacto clínico y los riesgos asociados con anemia después de HDA. Se ha demostrado que los pacientes con valores de $\mathrm{Hb}<10$ $\mathrm{g} / \mathrm{dl}$ tenían un riesgo dos veces mayor de resangrado y mortalidad que los pacientes con $\mathrm{Hb} \geq 10 \mathrm{~g} / \mathrm{dl}$. Claramente estos pacientes se beneficiarian de una suplementación con hierro ${ }^{(48)}$.

\section{ENFERMEDAD HEPÁTICA}

La anemia es común en la enfermedad hepática crónica y se caracteriza por su etiologia multifactorial, incluyendo deficiencia de ácido fólico, hiperesplenismo, hemodilución, hemólisis, supresión de la médula ósea por virus o alcohol, insuficiencia renal y la causa más importante, hemorragia gastrointestinal. La prevalencia varía según la etapa de la enfermedad, siendo reportada \% de la cirrosis hepática compensada, 50$70 \%$ de la descompensada y $67 \%$ en los pacientes con hepatocarcinoma ${ }^{(49)}$.
El hígado desempeña un papel importante en la homeostasis del hierro. Es el órgano principal para la producción de la hormona reguladora del hierro: hepcidina. Los niveles bajos de hepcidina en las enfermedades hepáticas, con o sin cirrosis, son probablemente uno de los factores que contribuyen a la anemia por enfermedad crónica presente en una variedad de afecciones hepáticas(50).

Las enfermedades hepatocelulares agudas y crónicas generalmente se asocian con coagulación sanguinea defectuosa debido a varias causas: disminución de la sintesis hepática de los factores II, VII, IX yX, la presencia de inhibidores de estos factores, trombocitopenia, función plaquetaria deteriorada, hiperfibrinolisis, y coagulación intravascular diseminada. Los defectos de coagulación que complican la enfermedad hepática predisponen a la hemorragia y AF, lo que aumenta tanto la morbilidad como la mortalidad(51).

La hemorragia aguda en estos pacientes puede ocurrir por várices esofágicas-gástricas secundarias a hipertensión portal o úlceras pépticas, frecuentes en pacientes con cirrosis. Las fuentes de hemorragia crónica en el paciente con patología hepática incluyen la ectasia vascular antral y gastropatía por hipertensión portal(50, 52).

La esplenomegalia, que generalmente es causada por hipertensión portal, puede provocar hemólisis secundaria, aumento en el volumen plasmático y macrocitosis. Puede observarse también anemia hemolítica debido a la destrucción intraesplénica de eritrocitos ${ }^{(53)}$.

En algunos pacientes, la insuficiencia de la médula ósea y la anemia aplásica se desarrollan después de un episodio de hepatitis. La aplasia severa de la médula puede ser inducida por los virus de las hepatitis B, C y también por otros, como el de Epstein-Barr y el de la inmunodeficiencia humana. Se ha postulado que a través de la mediación del interferón o la cascada de citoquinas, los virus inducen la activación de los linfocitos y finalmente la muerte apoptótica de las células hematopoyéticas. Las dos opciones principales para tratar la aplasia medular inducida por hepatitis son el trasplante de células hematopoyéticas y la terapia inmunosupresora ${ }^{(54)}$.

En pacientes con hepatitis C crónica, la anemia es una complicación del tratamiento con Ribavirina. La hemólisis inducida se puede revertir al reducir la dosis del medicamento o suspenderlo por completo(50).

Finalmente, el alcohol como factor etiológico común en la cirrosis, es tóxico para la médula ósea. La toxicidad directa del alcohol causa la supresión reversible de la hematopoyesis y posteriormente, anemia con producción y función de plaquetas deterioradas. La mayoría de los pacientes con abuso crónico de alcohol 
también presentan deficiencias carenciales de hierro, ácido fólico, vitamina B12 y B6 ${ }^{(55)}$.

El diagnóstico de anemia en la enfermedad hepática crónica es muy desafiante, ya que los métodos de laboratorio, que incluyen hierro sérico, ferritina, saturación de transferrina y volumen corpuscular medio se ven afectados por la enfermedad o su causa. Se están estudiando nuevos parámetros para identificar una deficiencia verdadera de hierro en estos pacientes: ferritina de glóbulos rojos, prueba e índice del receptor de transferrina sérica y hepcidina ${ }^{(49)}$.

El manejo terapéutico inicial se basa en identificar y tratar la etiología y reponer la anemia. En pacientes con cirrosis hepática, el objetivo debe ser la prevención y el tratamiento de las complicaciones de la hipertensión portal, con betabloqueantes no selectivos y terapia endoscópica (ligadura de las VE). En la ectasia vascular antral, es frecuentemente la falta de respuesta a los tratamientos que reducen la presión portal y el tratamiento se basa en técnicas endoscópicas de coagulación con gas argón ${ }^{(54)}$.

La segunda parte del manejo terapéutico es restaurar los depósitos de hierro y normalizar los indices hematimétricos. La forma más conveniente y efectiva de lograrlo es la prescripción de hierro por vía oral siempre que el paciente esté estable. En pacientes cirróticos se debe tener precaución debido a su menor absorción y riesgo de encefalopatía hepática a consecuencia de estreñimiento secundario a su uso. Para aquellos que son intolerantes o no responden al hierro oral, se administra hierro i/v. La transfusión de sangre debe reservarse para pacientes que son inestables o que permanecen sintomáticos a pesar de la terapia con hierro. Éstas no apuntan a un nivel normal de $\mathrm{Hb}$, sino a un nivel seguro (para evitar el incremento de la presión portal)(49).

\section{PÓLIPOS Y CÁNCER COLORRECTAL}

La presencia de anemia y la deficiencia de hierro son un hallazgo frecuente en el CCR, con prevalencia de $50 \%$ a $60 \%$. Es consecuencia de las pérdidas sanguíneas endoluminales provocadas por el tumor. Generalmente ésta se presenta de manera asintomática hasta estadios avanzados de la enfermedad. Los factores de riesgo de anemia en el CCR son el mayor diámetro del tumor y la localización en el colon derecho(56).

La anemia en el CCR se ha asociado a peor pronóstico y reducción en la sobrevida. Un estudio, realizado en Uruguay con 411 pacientes evidenció que la presencia de anemia en pacientes con CCR tiene incidencia negativa en el pronóstico, aumentando tanto la mortalidad global como quirúrgica. Por lo que se sugiere tratamiento adecuado e individualizado en el preoperatorio $(57)$
Los pólipos colorrectales son lesiones frecuentes, peerse no son causa de anemia. En ocasiones las complicaciones dadas por el gran tamaño, erosiones y microsangrados, podrian justificar una AF. De esto se infiere que no se debería atribuir la anemia a pólipos menores de $10 \mathrm{~mm}$.

\section{CIRUGIAS GASTROINTESTINALES}

Las técnicas de cirugía bariátrica se dividen en tres grupos, técnicas restrictivas que sirven para limitar la ingesta de alimentos, malabsortivas que interfieren con el proceso digestivo normal y mixtas que comparten caracteristicas de los dos grupos anteriores.

En el período postoperatorio se estima que $20-30 \%$ de los pacientes presentan anemia y hasta 50\% ferropenia. Estas pueden deberse a múltiples causas como descenso de la ingesta, disminución de proteasas e hipoclorhidria y déficit de factor intrinseco(58)

La úlcera marginal también puede ser causa de anemia crónica o hemorragia digestiva en el paciente sometido a cirugia bariátrica. Existen dos tipos, la precoz que es la más frecuente y se presenta en las primeras 48 horas post cirugía, y la tardía. Generalmente se localiza en la vertiente yeyunal de la anastomosis gastroyeyunal. Los factores relacionados a presencia de la úlcera son la estenosis del estoma, material de sutura en el local de la anastomosis, tabaquismo, infección por H.pylori y consumo de AINE(59).

Los pacientes sometidos a una cirugía bariátrica malabsortiva deben recibir suplementación con hierro de por vida. Además, debe considerarse la administración de otros nutrientes, como vit B12 y ácido fólico. La deficiencia de hierro y la anemia pueden desarrollarse años después de la cirugía. En pacientes con bypass gástrico, el duodeno y yeyuno proximal quedan excluidos por lo que la reposición con hierro I/V es la mejor opción.

En otro tipo de gastrectomías se produce malabsorción de hierro por disminución de la producción de ácido clorhídrico. También pueden verse úlceras en la anastomosis y riesgo aumentado de cáncer gástrico postgastrectomía tanto en la cirugía realizada por patología benigna como maligna.

Las resecciones intestinales también son causa de anemia por malabsorción de hierro, vit B 12 y ácido fólico. El tipo y grado de malabsorción dependen de diversos factores como la longitud y sector de intestino resecado, asi como la capacidad de adaptación intestinal. En general se toleran peor las resecciones de ileon que las de yeyuno. Las resecciones ileales de más de $60 \mathrm{~cm}$ producen habitualmente malabsorción de vit B12 clinicamente sintomática(60).

El sindrome de intestino corto (intestino delgado distal al duodeno $\leq 150-200 \mathrm{~cm}$ ). Y la insuficiencia 
intestinal (reducción de la función intestinal por debajo del mínimo necesario para la absorción de macronutrientes, agua y electrolitos) son entidades donde es necesaria la suplementación parenteral de hierro, vit B12 y ácido fólico(61, 62)

\section{CONCLUSIONES}

La anemia plantea un desafío diagnóstico. Su causa suele ser multifactorial. El sangrado, malabsorción e inflamación juegan un papel importante en el contexto de diferentes afecciones gastrointestinales. Puede contribuir sustancialmente a la morbilidad y mortalidad de un trastorno subyacente, reducir la calidad de vida, la capacidad de ejercicio y la función cognitiva.

Corregir la causa de la anemia o la anemia en si cuando la causa no pueda tratarse son las claves del tratamiento

\section{REFERENCIAS}

1. Organizacion de las Naciones Unidas para la Alimentación y la Agricultura, Organización Panamericana de la Salud. Panorama de la seguridad alimentaria y nutricional. Santiago: FAO, 2017

2. World Health Organization. Worldwide Prevalence of Anaemia. WHO, 2008; 1: 1993-2005.

3. Looker A, Dallman P, Carroll M, Gunter E, et al. Prevalence of iron deficiency in the United States. JAMA, 1997; 277: 973-76.

4. Sans J, Besses C, Vives J. Hematología clínica: La anemia, aspectos generales del diagnóstico. Madrid, Elsevier España; 2006: 107-126.

5. Kasi M, Bowling T. Anemia in gastroenterology. Medicine, 2015; 12 : 8-10.

6. McIntyre A, Long R. Prospective survey of investigations in outpatients referred with iron deficiency anaemia. Gut, 1993 34: 1102-7.

7. Weiss G, Ganz T, Goodnough L. Anemia of inflammation. Blood, 2019; 40: 133

8. Moreno J, Romero $\mathbf{M}$, Gutierrez $\mathbf{M}$. Classification of anemia for gastroenterologists. World J Gastroenterol, 2009; 15(37): 4627-37.

9. Ko CW, Siddique SM, Patel A, Harris A, Sultan S, Altayar O Falck-Ytter Y. AGA Clinical Practice Guidelines on the Gastrointestinal Evaluation of Iron Deficiency Anemia. Gastroenterology 2020:159(3):1085-1094.

10. Rizvi S, Schoen R. Supplementation with oral vs intravenous iron for anemia with IBD or gastrointestinal bleeding Isoraliron getting abadrap?. AmJGastroenterol, 2011; 106: 1872-9.

11. Anderson G, Frazer D, McLaren G. Iron absorption and metabolism. CurrOpinGastroenterol, 2009; 25: 129-35.

12. Jolobe $\mathbf{O}$. Prevalence of hypochromia vs microcytosis in iron deficiency. Clin Lab Haematol, 2000; 22: 79-80.

13. Nicolas G, Viatte L, Bennoun M, Beaumont C, Kahn A, Vaulont S Hepcidin, a new iron regulatory peptide. Blood Cells Mol Dis, 2002; 29: 327-35

14. Goddar A, James M , McIntyre A, Scott B. Guidelines for the management of iron deficiency anaemia. Gut, 2011; 60: 1309160.

15. Green B, Rockey C. Gastrointestinal endoscopic evaluation of premenopausal women with iron deficiency anemia. 104-9, s.l. : JClin Gastroenterol, 2004, Vol. 38

16. Rodriguez L, Rodriguez F, Ruiz A, Loba T, Botargues J, Guardiola J. Nuevo abordaje en el estudio de anemia ferropénica basado en el test de sangreo culto en heces inmunológico cuantitativo. GastroenterolHepatol, 2013; 36: 151.

17. Tee H, Kaffes A. Non small bowel lesions encountered duringdouble balloon enteroscopy performed for obscure gastrointestinal bleeding. World J Gastroenterol, 2010; 16: 1885-9.

18. Gilbert D, O'Malley S, Selby W. Are repeat upper gastrointestinal endoscopy and colonoscopy necessary within six months of capsule endoscopy in patients with obscure gastrointestinal bleeding?. J Gastroenterol Hepatol, 2008; 23: 1806 - 9.

19. Koulauzidis A, Rondonotti E, Giannakou A, Plevris J. Diagnostic yield of small-bowl capsule endoscopy in patients with irondeficiency anemia: a systematic review. Clinical Endoscopy, 2012; 76(5): 983-92

20. Pennazio M, Santucci R, Rondonotti E, Abbiati C, Beccari G, Rossini F. Out come of patients with obscure gastrointestinal bleeding after capsule endoscopy: Report of 100 consecutive cases. Gastroenterology, 2004; 126: 643-48.

21. Godeschalk M, Mensink B, VanBuuren H, Kuipers E. Primary balloon assisted enteroscopy in patients with obscure gastrointestinal bleeding: Findings and out come of therapy. JClin Gastroenterol, 2010; 44(1): 195-20.

22. Rimon E, Kagansky N, Kagansky M, Mechnic L, Mashiah T, Namir $\mathbf{M}$, et al. Are wegiving too much iron? Low dose iron therapy is effective in octogenarians. AmJMed, 2005; 118: 1142-7.

23. De Silva A, Tsironi E, Feakins R, Rampton D. Efficacy and tolerability of oral iron therapy in inflammatory bowel disease: A prospective, comparative trial. AlimentPharmacolTher, 2005; 22:1097-105.

24. Silverstein S, Rodgers G. Parenteral iron therapy options. Am J Hematol, 2004; 76: 74-78.

25. Lyseng K, Keating G. Ferric carboxymaltose: A review of it suse in iron deficiency anaemia. Drugs, 2009; 69: 739-56.

26. Jeffrey L, Carson M, Gordon G, Nancy M, et al. Clinical Practice Guidelines From the AABB Red Blood Cell Transfusion Thresholds and Storage. JAMA, 2016; 316(19): 2025-35.

27. Burda P, Kuster A, Hjalmarson O, Suormala T, Bürer C, Lutz S, et al. Characterization and review of MTHFD1 deficiency: four new patients, cellular delineation and response to folic and folinic acid treatment. J Inherit Metab Dis, 2015; 38(5): 835-72.

28. Toh B. Diagnosis and classification of autoimmune gastritis. Autoimmun Rev, 2014; 13(4-5): 459-62.

29. Hariz A, Bhattacharya P. Megaloblastic Anemia. StatPearls Publishing, 2020. https://www.ncbi.nlm.nih. gov/books/ NBK537254

30. Green R. Vitamin B12 deficiency from the perspective of a practicing hematologist. Blood, 2017; 129(19): 2603-11.

31. Sanz T, González P, Riesgo R, Garrido S, del Cura I, Martín J, et al. Oral versus intramuscular administration of vitamin B12 for the treatment of patients with vitamin B12 deficiency. BMC Public Health, 2012; 12: 394.

32. Moscoso F, Quera R. Enfermedad celiaca. Revisión. Rev. méd. Chile, 2016; 144( 2 ): 211-221.

33. Martín R, Nestares M, Díaz J, López I, Muñoz MJ, Moreno J, et al. Multifactorial Etiology of Anemia in Celiac Disease and Effect of Gluten-Free Diet: A Comprehensive Review. Nutrients, 2019: 2557.

34. Abu-Daya H, Lebwohl B, Lewis S, Green P. Celiac disease patients presenting with anemia have more severe disease than those presenting with diarrhea. Clin Gastroenterol Hepatol, 2013; 11: 1472-7.

35. Grisolano S, Oxentenko A, Murray J, Burgart L, Dierkhising R, Alexander J. The usefulness of routine small bowel biopsies in evaluation of iron deficiency anemia. Clin Gastroenterol, 2004: 38(9): 756-60.

36. Wierdsma N. Vitamin and Mineral Deficiencies Are Highly Prevalent in Newly Diagnosed Celiac Disease Patients. Nutrients, 2013: 5: 3975-92.

37. Garcia Á, Lucendo A. Review: Nutritional and Dietary Aspects of 
Celiac Disease. Nutr. Clin. Pract, 2011; 26: 163-173.

38. Berry N, Basha J, Varma N, Varma S, Kishor K, Vaiphei K. Anemia in celiac disease is multifactorial in etiology: A prospective study from India. JGH Open, 2018; 2(5): 196-200.

39. Kulnigg S, Gasche C. Systematic review: Managing anaemia in Crohn's disease. Aliment Pharmacol Ther, 2006; 24: 1507-23.

40. De la Morena F, Gisbert J. Anemia and inflammatory bowel disease. Rev Esp Enferm Dig , 2008; 100: 285-293.

41. Gasche C, Berstad A, Befrits R, Beglinger C, Dignass A, Erichsen K, Gomollon F, et al. Guidelines on the diagnosis and management of iron deficiency and anemia in inflammatory bowel diseases. Inflamm Bowel Dis, 2007; 13: 1545-53.

42. Gomollón F, Gisbert J. Current management of iron deficiency anemia in inflammatory bowel diseases: a practical guide. Drugs, 2013: 73: 1761-1770.

43. Dignass A, Gasche C, Bettenworth D, Birgegård G, Danese S, Gisbert J, et al. European Crohn's and Colitis Organisation [ECCO]. European consensus on the diagnosis and management of iron deficiency and anaemia in inflammatory bowel diseases. J Crohns Colitis, 2015; 9: 211-22.

44. Kulnigg S, Teischinger L, Dejaco C, Waldhor T, Gasche C. Rapid recurrence of IBD-associated anemia and iron deficiency after intravenous iron sucrose and erythropoietin treatment. Am J Gastroenterol, 2009; 104: 1460-7.

45. Miilunpohja S, Jyrkkä J, Kärkkäinen J, Kastarinen H, Heikkinen M, Paajanen $\mathbf{H}$ et al. Long-term mortality and causes of death in endoscopically verified upper gastrointestinal bleeding: comparison of bleeding patients and population controls. Scand J Gastroenterol, 2017;(12): 1-8

46. Oakland K, Guy R, Uberoi R, Hogg R, Mortensen N, Murphy M, Jairath V. UK Lower Gl Bleeding Collaborative. Acute lower Gl bleeding in the UK: patient characteristics, interventions and outcomes in the first nationwide audit. Gut, 2017; 313-428.

47. Bager P, Dahlerup JF. Lack of follow-up of anaemia after discharge from an upper gastrointestinal bleeding centre. Dan Med J. 2013: 60: A4583

48. Rockall T, Logan R, Devlin H, Northfield T. Risk assessment after acute upper gastrointestinal haemorrhage. Gut, 1996; 38(3): 16-321.

49. Gonzalez R, Jones E , Moreno R. Spectrum of anemia associated with chronic liver disease World J Gastroenterol, 2009; 15(37): 4653-58

50. Gkamprela E, Deutsch M, Pectasides D. Iron deficiency anemia in chronic liver disease: etiopathogenesis, diagnosis and treatment. Ann Gastroenterol, 2017; 30(4): 405-413.

51. Thachil J. Anemia - The overlooked factor in bleeding related to liver disease. J Hepatol, 2011; 54(3): 593-4.

52. Amitrano L, Guardascione M, Brancaccio V, Balzano A. Coagulation disorders in liver disease. Semin Liver Dis, 2002; 22: 83-96.

53. Goddard A, James M, McIntyre A, Scott B. Guidelines for the management of iron deficiency anaemia. British Society of Gastroenterology. Gut, 2011 Oct; 60(10):1309-16.

54. Ong J, Younossi Z. Managing the hematologic side effects of antiviral therapy for chronic hepatitis C: anemia, neutropenia, and thrombocytopenia. Cleve Clin J Med, 2004; 64: 17-21.

55. loannou G, Dominitz J, Weiss N, Heagerty P, Kowdley K. The effect of alcohol consumption on the prevalence of iron overload, iron deficiency, and iron deficiency anemia. Gastroenterology, 2004. 126(5): 1293-301.

56. Ludwig H, Müldür $E$, Endler $G$, Hübl W. Prevalence of iron deficiency across different tumors and its association with poor performance status, disease status and anemia. Ann Oncol 2013;24(7):1886-1892

57. Della Valle A, Sarroca C. Importancia de la anemia en el cáncer colorrectal. Rev Med Uruguay 2004; 20: 114-119

58. Stein J, Stier C, Raab H, Weiner R. Review article: The nutritional and pharmacological consequences of obesity surgery. Aliment Pharmacol Ther, 2014; 40: 582-609.

59. Aills L, Blankenship J, Buffington C, Furtado M, Parrott J. ASMBS
Allied Health Nutritional Guidelines for the Surgical Weight Loss Patient. Surg Obes Relat Dis, 2008; 4: 73-108.

60. Green R, Datta Mitra A. Megaloblastic Anemias: Nutritional and Other Causes. Med Clin North Am. 2017 Mar; 101(2):297-317.

61. Billiauws L, Maggiori, Joly F, Panis Y. Medical and Surgical Management of Short Bowel Syndrome. J Visc Surg. 2018 Sep; 155 (4): 283-291.

62. Donohoe CL, Reynolds JV. Short Bowel Syndrome. Surgeon. 2010 Oct; 8(5):270-9.

\section{Abreviaturas}

AF: anemia ferropénica

AINEs: antinflamatorios no esteroideos

Hb: hemoglobina

CE: cápsula endoscópica

CV: cardiovascular

EC: enfermedad celiaca

Ell: enfermedad inflamatoria intestinal

HDA: hemorragia digestiva alta

HDB: hemorragia digestiva baja

HDM: hemorragia digestiva media

TGI: tracto gastrointestinal

VGC: videogastroscopia

VCC: videocolonoscopia

VE: varices esofágicas

Vit B12: vitamina B12

Nota del autor:

Los autores no declaran conflictos de in terés o soporte financiero.

\section{Nota de contribución:}

Todos los autores han participado en las diferentes etapas de elaboración de este trabajo, reúnen las condiciones de autoria y han aprobado la versión final del mismo.

\section{Nota del Editor:}

El presente manuscrito fue aprobado por Nelia Hernández como su editora.

Recibido: 14/02/2021

Aceptado: 02/03/2021 